\title{
Randomized trial of acupoints herbal patching in Sanfu Days for asthma in clinical remission stage
}

\author{
Libing Zhu ${ }^{1,3}$, Wei Zhang ${ }^{1}$, Vivian Wong ${ }^{1}$, Ziea Eric ${ }^{2}$, Lixing Lao ${ }^{1}$, Kwaiching Lo ${ }^{1}$, Waichung Chan ${ }^{1}$, To Yau ${ }^{1}$
} and Lei Li $i^{*}$

\begin{abstract}
Background: Although China has a long history of using acupoints herbal patching (acupoints herbal patching means applying herbal patch on special acupoints to stimulate skin to form blisters, hyperemia, and even suppuration) in Sanfu Days (Sanfu Days are supposed to be the three hottest days in a year which is calculated by the ancient calendar) for the treatment of asthma, there is insufficient evidence to support its effectiveness and safety issues. This study investigated the efficacy and safety of acupoints herbal patching compared with placebo in participants with asthma in clinical remission stage.
\end{abstract}

Methods: We enrolled participants with asthma in clinical remission stage, above 13 years old and both genders in a randomized, double-blind and placebo-control trial at clinical center, School of Chinese Medicine, The University of Hong Kong to assess the effectiveness and safety of acupoints herbal patching, as compared with placebo, when added to guidelines-based therapy. The trial was conducted for three times (these three times were 19 July, 29 July and 8 August 2010), and the primary outcome was pulmonary function test. Secondary outcome was self-made questionnaire which were designed based on Traditional Chinese Medicine theory and clinical experience summary.

Results: Three hundred and twenty three eligible participants were enrolled, they were randomly assigned to acupoints herbal patching group $(n=165)$, placebo control group $(n=158)$. There was no significant difference in primary and secondary outcome as compared with placebo group at the end of 3rd treatment and four times follow ups. But sub-analysis of secondary outcome in four times follow ups showed that acupoints herbal patching significantly reduced the proportion of participants who didn't need medical treatment when they had a small rise in asthma-related symptoms increased from 6-15\% at 1st follow up and 0-7\% at 3rd follow up (P < 0.05). It indicated that the proportion of participants who can spontaneous resolution of an asthma attack increased through acupoints herbal patching. In addition, acupoints herbal patching was significantly superior to placebo in reducing the percentage of participants who were susceptibly waken up by asthma symptoms from $27-14 \%$, and the percentage of participants who had the symptom of running nose and sneezing before onset from $18-8 \%$ at 2 nd follow up $(P<0.05)$. Improvements also occurred with treatment group, it reduced the proportion of participants who were spontaneous sweating at 3rd follow up $(P<0.05)$.

Conclusions: There was no significant difference between acupoints herbal patching and placebo in pulmonary function test in this study. Self-made questionnaire showed that the lasting effect of acupoints herbal patching was significantly better than placebo in reducing the need for medications to control asthma and the proportion of susceptible symptoms in participants with asthma in clinical remission stage. It showed that the low quality of life caused

\footnotetext{
*Correspondence: Ilie@hku.hk

${ }^{1}$ School of Chinese Medicine, The University of Hong Kong, 10 Sassoon

Road, Pokful, Hong Kong, China

Full list of author information is available at the end of the article
}

\section{贷 Springer}

(c) 2016 Zhu et al. This article is distributed under the terms of the Creative Commons Attribution 4.0 International License (http:// creativecommons.org/licenses/by/4.0/, which permits unrestricted use, distribution, and reproduction in any medium, provided you give appropriate credit to the original author(s) and the source, provide a link to the Creative Commons license, and indicate if changes were made. 
by asthma-related symptoms was significantly improved through acupoints herbal patching in Sanfu Days. Besides, acupoints herbal patching was as safe as placebo for chronic stable asthma.

Trial registration number: HKUCTR-1128, Registration date 22 Jul 2010

Keywords: Asthma, Randomized trial, Acupoints herbal patching, Sanfu days

\section{Background}

Asthma is a clinical syndrome of unknown etiology that diagnosed on the basis of symptoms including wheeze, dyspnoea, and cough, etc, accompany with the objective evidence of airflow obstruction [1]. Currently, $\beta$-agonists still as the first-line drug in treating acute asthma attack and glucocorticoids used to treat chronic asthma while are associated with various adverse effects [2-10]. In china, there was a long history of using acupoints herbal patching in Sanfu Days for preventing and treating asthma. Sanfu Days are calculated by Heavenly Stems and Earthy Branches which are supposed to be the three hottest days in a year. According to the provision, the third and fourth Gengri after the summer solstice is recorded as the first and second hottest days, and the first Gengri after the beginning of autumn is recorded as the third hottest day. In these days, body's Yang-qi (positive energy) has an exuberant trend with the aid of Yang-qi developed by nature, and the Cold-qi in the body also in the condition of easily dissolve. Acupoints herbal patching means applying medicinal cakes which are made of Chinese herbs on special acupoints. These herbs are pungent in flavor, warm in property, and have the function of dredging the channel, which can stimulate skin to form blisters, hyperemia and even suppuration. As a result, acupoints herbal patching combined with Sanfu Days get a purpose of adjusting Yin and Yang, preventing and curing diseases, and enhancing body resistance through acupoints stimulation and drug infiltration absorption. In modern terms, acupoints herbal patching is similarly to transdermal drug delivery. Many Chinese scientists have already conducted some animal and clinical studies on the efficacy of acupoints herbal patching for the treatment of asthma. So far, they found that acupoints herbal patching was effective in improving lung function and syndromes in participants with asthma not only from animal studies but also clinical studies [11-19]. But the weakness of previous clinical studies, such as poor methodologically, small sample size, short follow-up, didn't mention whether get Institutional Review Board (IRB) approval before study, also not mentioned dropout rate and safety issue, all these make people unconvinced of the previous findings [20-22]. Hence, this study tried to design a more rigorous, bigger sample size and longer follow-up clinical randomized control trial (RCT) aim to investigate the efficacy and safety of acupoints herbal patching compared with placebo. The hypothesis of this study was that there was no significant difference between acupoints herbal patching group and placebo group in preventing and reducing the frequency and severity of asthmatic attacks.

\section{Methods}

\section{Participants}

This study was a randomized, double-blind, placebocontrolled trial in 323 asthma participants in clinical remission stage (above 13 years old and in both genders). The diagnose standard of asthma in clinical remission stage means participants had a history of asthma attack in the past 12 months, but asthma-related signs and symptoms disappeared, pulmonary function recover to the level before acute asthma attack and maintain more than 3 months when they entered this study. Documentation of symptoms of asthma or a physician's diagnosis of asthma for more than 1 year preceding the study visit was required. Participants need to meet the inclusion criteria listed as follow: symptoms occurring or worsening at night, episodic symptoms of airflow obstruction; difficulty breathing, chest tightness, cough (worse at night); symptoms awakening the patient, occurring or worsening with exercise; viral infections, and changes in weather.

Participants would be ineligibility if they meet one or more following exclusion criteria: acute asthma attack, fever and pharyngitis, pregnancy, tuberculosis, severe cardiac and pulmonary diseases. Participants were also excluded if they had diabetes mellitus, allergy to topical medication and hypersensitive skin condition. Additional exclusion criteria were bleeding disorders, severe heart diseases or with pacemaker and keloid.

The study protocol (21006030HA) was approved by the Institutional Review Board of the University of Hong Kong/Hospital Authority Hong Kong West Cluster (HKU/HA HKWC IRB) with coding UW 10-274. This clinical trial was authorized by Clinical Trials Centre of The University of Hong Kong (HKUCTR-1128). URL of register can be found in http://www.hkuctr.com/Study/ Show/f5d04b243cb14c25b9033ec55e96f6f5. Grants from Hospital Authority (No.200006030). All study participants need to provide written informed consent, those younger than 18 years of ages were also required to provide assent and then signature by their parent or legal guardian. 


\section{Procedures}

After screening visit, enrolled participants were randomly assigned to an acupoints herbal patching group and a placebo control group by a third person (Wang ZH) and she did not practice throughout the study. Random numbers were distributed by the formula of [= rand ()] in Microsoft Office Excel 2007 (fraction between 0 and 1 ), and if the produced random number $\geq 0.5$, it was assigned to acupoints herbal patching group, and otherwise, it was belonged to placebo group. The principal investigator of this study-Dr. Li L, who obtained Ph.D. degree of acupuncture and moxibustion and have more than 30 years acupuncture clinical experience. Besides he is not only the registered Chinese Medicine practitioner in Mainland China, but also Hong Kong registered Chinese Medicine practitioner. The rest practitioners who participated in this study all have bachelor or bachelor above degree of Traditional Chinese Medicine (TCM), and all of them are registered Chinese Medicine practitioners.

A study coordinator (Choi PY) allocated the random numbers to participants. Regarding as the allocation results, they were sealed into opaque envelopes and allocation concealment must be warranted. Totally twelve medical beds were provided during these three treatment days and treatment hours were from 9 a.m. to 7 p.m. During the treatment days, one usher (totally ten ushers who were Li XZ, Ching WT, Yau T, Zhang WD, Tse WM, Wu TT, Kan WY, Lo MF, Shi HF and Irene) guided one patient to the reception room, and then the receptionist (totally two receptionist: Leung LF and Lam CK) checked the random numbers list to decide which kind patch was given for this participant and told the result to taking patch person (totally two taking patch person: Lam WC and Chia KL). Then taking patch person took a sealed box with eleven patches inside to plaster (totally 10 plasters: Li L, Chan WC, Lo KC, Zhang W, Eric Z, Wong V, Tin PY, Chan HN, Cheung SK and Choi WF). Plaster spent nearly 5 min to paste patches in acupoints (totally 11 acupoints, and one patch for one acupoints), and then plaster used approximately $4 \mathrm{~cm} \times 4 \mathrm{~cm}$ hypoallergenic tape $\left(3 \mathrm{M}^{\mathrm{TM}}\right.$ Micropore $^{\mathrm{TM}}$ Tape $\left.1535-3\right)$ to stick patches in skin. Then the usher took the participant to waiting room, research assistants (Wu FM and Cheung CY) in waiting room removed the patches after $2 \mathrm{~h}$. Last, participants can leave treatment place if they didn't have any uncomfortable. During the whole process, nobody has idea of which treatment they have been received or given except Wang ZH. Until final statistic finished, Wang ZH managed unblinding and the random code was broken.

Both the acupoints herbal patching and placebo treatment was consisted of three sessions with $2 \mathrm{~h}$ duration (these three sessions were 19 July, 29 July and 8 August
2010). For all participants, 11 acupoints were applied by patches: Du14, Du12, Du4, BL13 (both side), BL23 (both side), BL43 (both side) and EX-B1 (both side) (Fig. 1). In both groups, every selected acupoints was applied one patch and every patch was about $2 \mathrm{~g}$ (Fig. 2). The final formula used in treatment group was a combination of the record in the "Zhang' Medicine" and the formula frequency used in clinical trials. In the acupoints herbal patching group, the selected herbals were Sinapi Alba, Radix Corydalis Yanhusuo, Processed Euphorbia kansui, Asari Herba cum Radice, Ephedrae Herba, Processed Radix Aconiti Praeparata, Cinnamomum cassia and Eugenia caryophyllata, with a proportion in 2:1:1:1:1:1:1:1. At last, these herbals were mixed together with ginger juice. In placebo group, the selected materials with a proportion of 3:3:1 including pure starch, red rice and black glutinous rice, which finally were mixed together by petroleum jelly (Fig. 3).

All participants completed the self-made questionnaires and pulmonary function test at baseline, the end of 3rd treatment, and four times follow ups (Fig. 4). Questionnaires were assessed by physicians ( $\mathrm{Li} \mathrm{L}$, Chan
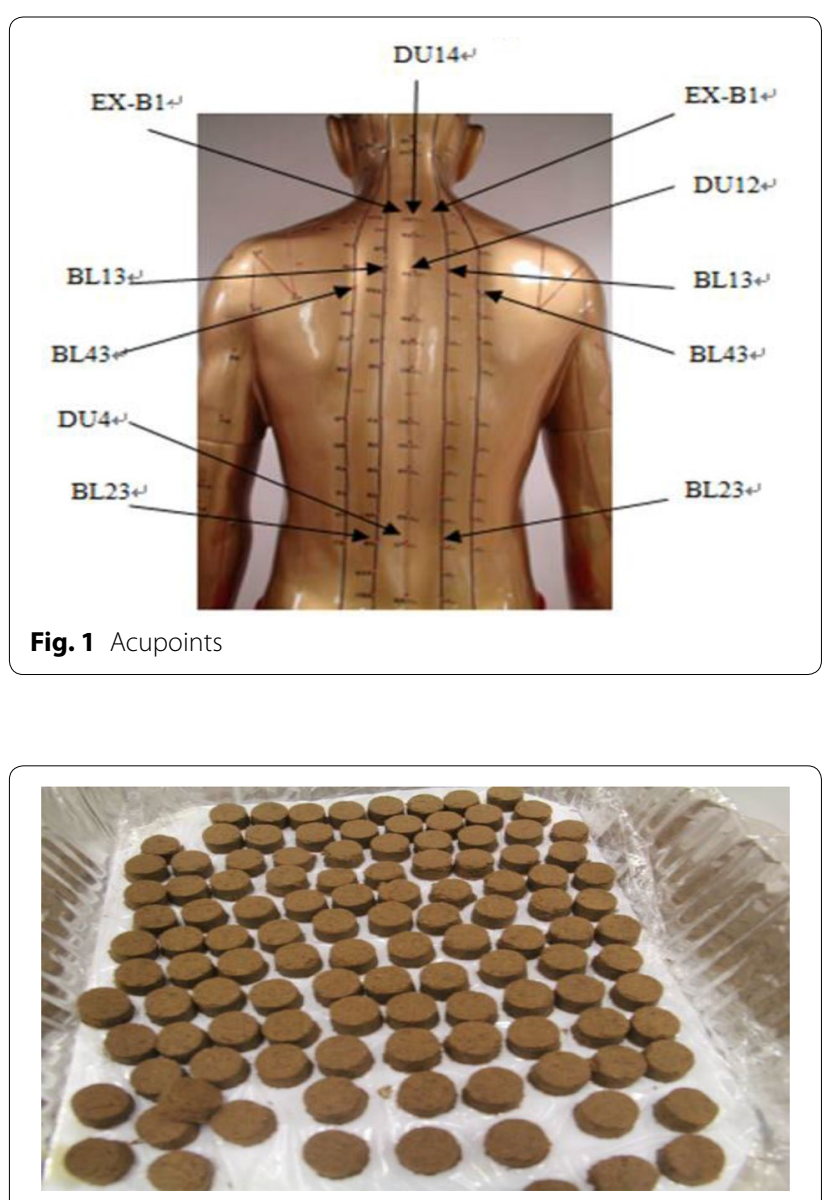

Fig. 2 Herbal patch 


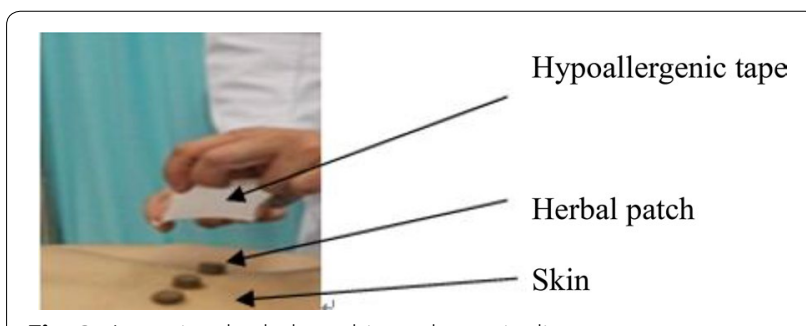

Fig. 3 Acupoints herbal patching schematic diagram

WC, Lo KC, Zhang W, Eric Z, Wong V, Tin PY, Chan HN, Cheung SK and Choi WF) through face to face. The primary outcome was pulmonary function test: forced expiratory volume in one second (FEV1) and forced expiratory volume in one second/forced vital capacity [FEV1/FVC (\%)], the pulmonary function test machines were provided by the lab in School of Chinese Medicine, The University of Hong Kong and all operators didn't know they do lung function test for which group participants. Secondary outcome was self-made questionnaire which included the number of days with asthma-related symptoms, asthma-related health care use, the frequency of bronchodilator used during asthma attack, the number of asthma related symptoms which were associated with Chinese Medicine and the percentage of participants with twenty-three specific such symptoms. These twenty-three symptoms were shown in Table 2.

\section{Statistical analysis}

The participants who received at least baseline assessment constituted the intention-to-treat (ITT) analysis, with a two-sided alpha level of 0.05 . For all primary and secondary outcome measures, sensitivity analyses were performed by replacing missing data with the last value. An additional per-protocol analysis (PP) was carried out for participants without major protocol violation who could be assessed at the end of 3rd treatment. Statistical analyses were performed with the Statistical Package for the Social Sciences (SPSS) software program (version 19) for Windows XP. The analysis included data from week 0 (baseline) through week 8 (at the end of 3rd treatment) and was performed with the use of an analysis of covariance (ANCOVA), group (acupoints herbal patching group and placebo group) as a fixed factor and time as covariance (baseline as the 1st time, post-treatment as the 2nd time). ANCOVA was conducted for continuous variables such as the number of asthma related symptoms which were associated with Chinese Medicine, the number of days with asthma-related symptoms and pulmonary function test: FEV and FEV1/FVC (\%) [23]. Binary logistic regression was conducted for the categorical variable such as asthma-related health care and the percentage of participants with twenty-three specific such symptoms [24]. Non-parametric test was conducted for a ranked variable such as the frequency of bronchodilator used during asthma attack. For the 1st, 2nd 3rd and
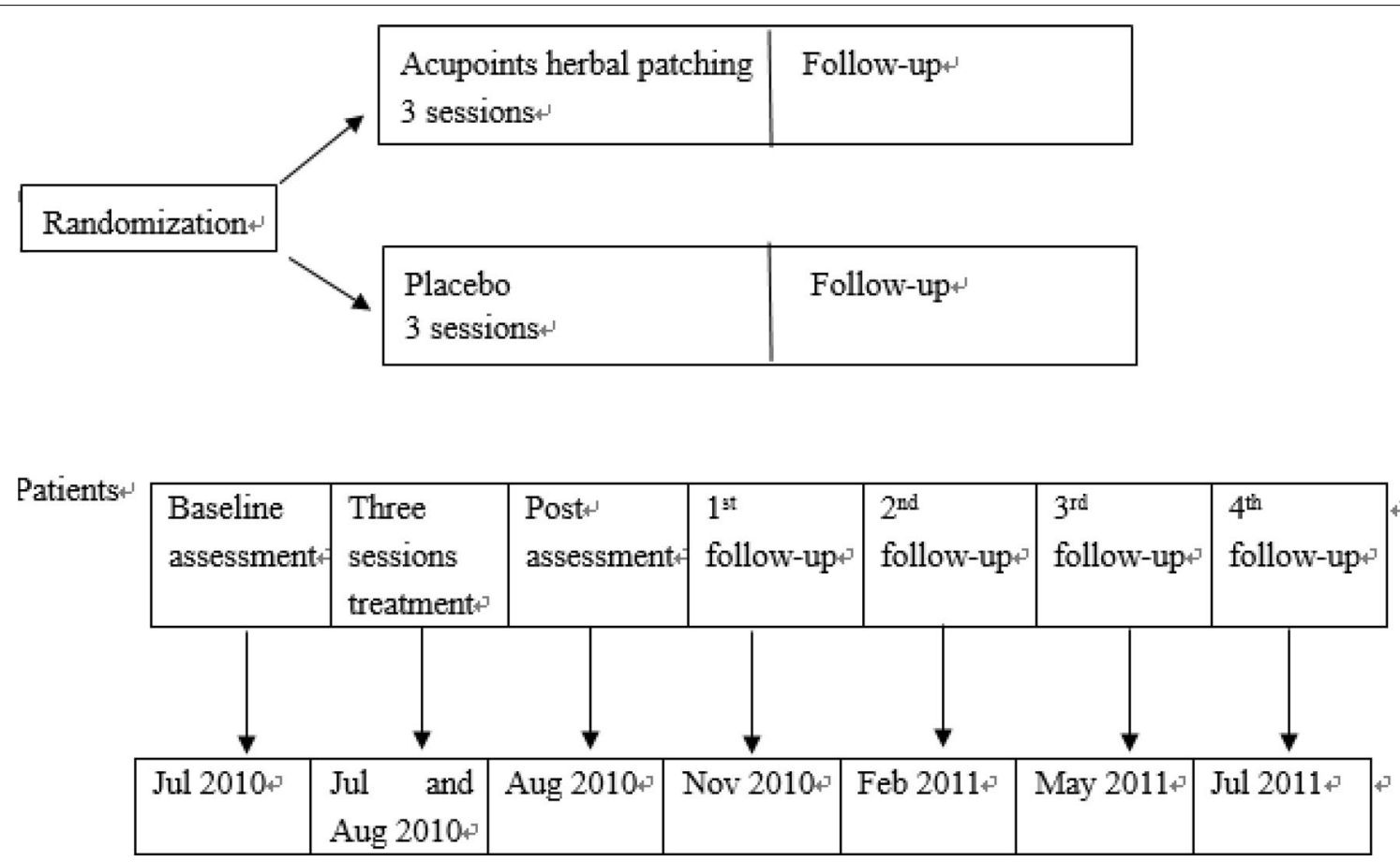

Fig. 4 Study design 
4th follow up measurements, two-sided $t$ tests and Chisquare tests were done for groups' pair wise comparisons [23]. Sample size was calculated according to the effective rate that conducted by previous clinical trials of acupoints herbal patch for asthma. The no. of target subjects to be recruited at local site was 380 (190 per treatment group $\times 2$ groups) through statistic.

\section{Results}

\section{Enrollment}

Initially, 451 participants with asthma in clinical remission stage applied to participate in this project. Figure 5 shows the trial flow. After screened, 323 participants underwent randomization: 165 to acupoints herbal patching group and 158 to placebo group. All subjects (323) were included in the ITT population. Forty-two participants in acupoints herbal patching group and thirty-nine participants in placebo group stopped the treatment prematurely. A PP analysis was conducted for the 242 subjects who could be assessed at the end of the 3rd treatment.

Tables 1, 2 showed the baseline characteristics of participants in the two study groups and their characteristics were basically similar: for both groups, the average age was 44 years old, $44 \%$ were male, and the average disease course of asthma were $22.74 \pm 22.68$ year in placebo group and $21.13 \pm 21.08$ year in acupoints herbal patching group. At enrollment, regarding the number of days on which subjects had asthma-related symptoms in acupoints herbal patching group was $10.25 \pm 12.26$ and $7.97 \pm 10.94$ in the placebo group. The mean $( \pm \mathrm{SD}) \mathrm{FEV}_{1}$ was $2.13 \pm 1.08$ in acupoints herbal patching group and $2.07 \pm 1.00$ in placebo group, and the mean ratio of $\mathrm{FEV}_{1}$ to FVC was $85.97 \pm 13.85$ in acupoints herbal patching group and $86.25 \pm 13.93$ in placebo group. Regarding the health care used when they had a small rise in asthmarelated symptoms, no difference occurred in treatment group and control group. Besides, there was nothing different between acupoints herbal patching group and placebo group in the distribution of the frequency of bronchodilator used during asthma attack in previous 1 year. Additionally, the number of symptoms which were associated with Chinese Medicine was also nearly same $(9.12 \pm 4.28$ in TG, $9.22 \pm 4.18$ in CG). There was also nothing different in percentage of participants with twenty-three specific such symptoms between acupoints herbal patching group and placebo group.

\section{Response to intervention}

After three times acupoints herbal patching, as compared with placebo, no changes occurred in primary outcome at the end of the 3rd treatment and four times follow ups (Tables 1, 3). Also, there was no significant difference appeared in secondary outcome at the end of the 3rd treatment (Table 2). But sub-analysis of secondary outcome in the following four times follow ups was found that acupoints herbal patching was superior to placebo in improving certain symptoms (Table 4). The result of sub-analysis show that acupoints herbal patching significantly reduced the proportion of participants who didn't need medical treatment when they had a small rise in asthma-related symptoms increased from 6-15\% compared with placebo group at the 1st follow up (Nov), and increased the proportion from $0-7 \%$ at the 3rd follow up (May) (Fig. 6, all $\mathrm{P}<0.05$ ). In other words, the proportion of participants who can spontaneous resolution of an asthma attack increased through acupoints herbal patching. Additionally, acupoints herbal patching as compared with placebo group significantly reduced the percentage of participants who are susceptibly waken up by asthma symptoms from $27-14 \%$ at 2nd follow up (Feb) (Fig. 7, $\mathrm{P}<0.05)$. Similarly, acupoints herbal patching significantly reduced the proportion of participants who had the symptom of running nose and sneezing before onset from $18-8 \%$ at 2 nd follow up (Fig. $8, \mathrm{P}<0.05$ ). Improvements also occurred with acupoints herbal patching group, it reduced the proportion of participants who were spontaneous sweating at 3rd follow up and diarrhea after intake of oily food at 4th follow up (Figs. 9, 10, July, all $\mathrm{P}<0.05$ ). All these findings showed that the low quality of life that caused by asthma-related symptoms was significantly improved through acupoints herbal patching in Sanfu Days. The top four symptoms which improved mostly in asthma participants were: susceptibly waken up by asthma symptoms and running nose, sneezing before onset which were belong to Lung-qi-deficiency; spontaneous sweating and diarrhea after intake of oily food were belong to Spleen-qi-deficiency. It showed that acupoints herbal patching has a better effect for those participants who were Lung-qi-deficiency and mainly showed as susceptibly waken up by asthma symptoms and running nose, sneezing before onset, and Spleen-qi-deficiency which mainly showed as spontaneous sweating and diarrhea after intake of oily food. Last but not least, the results of PP analysis were consistently with the ITT analysis.

\section{Safety}

Cutaneous reaction such as itching, skin warm feeling, swollen, blisters or pain were seen in almost all participants from acupoints herbal patching group. Participants in placebo group only have minor skin redness during the treatment. All these cutaneous reaction disappeared once stopped treatment. Five severe adverse events reported in treatment group: three participants appeared asthma exacerbation, one subject tended to vomited blood and 


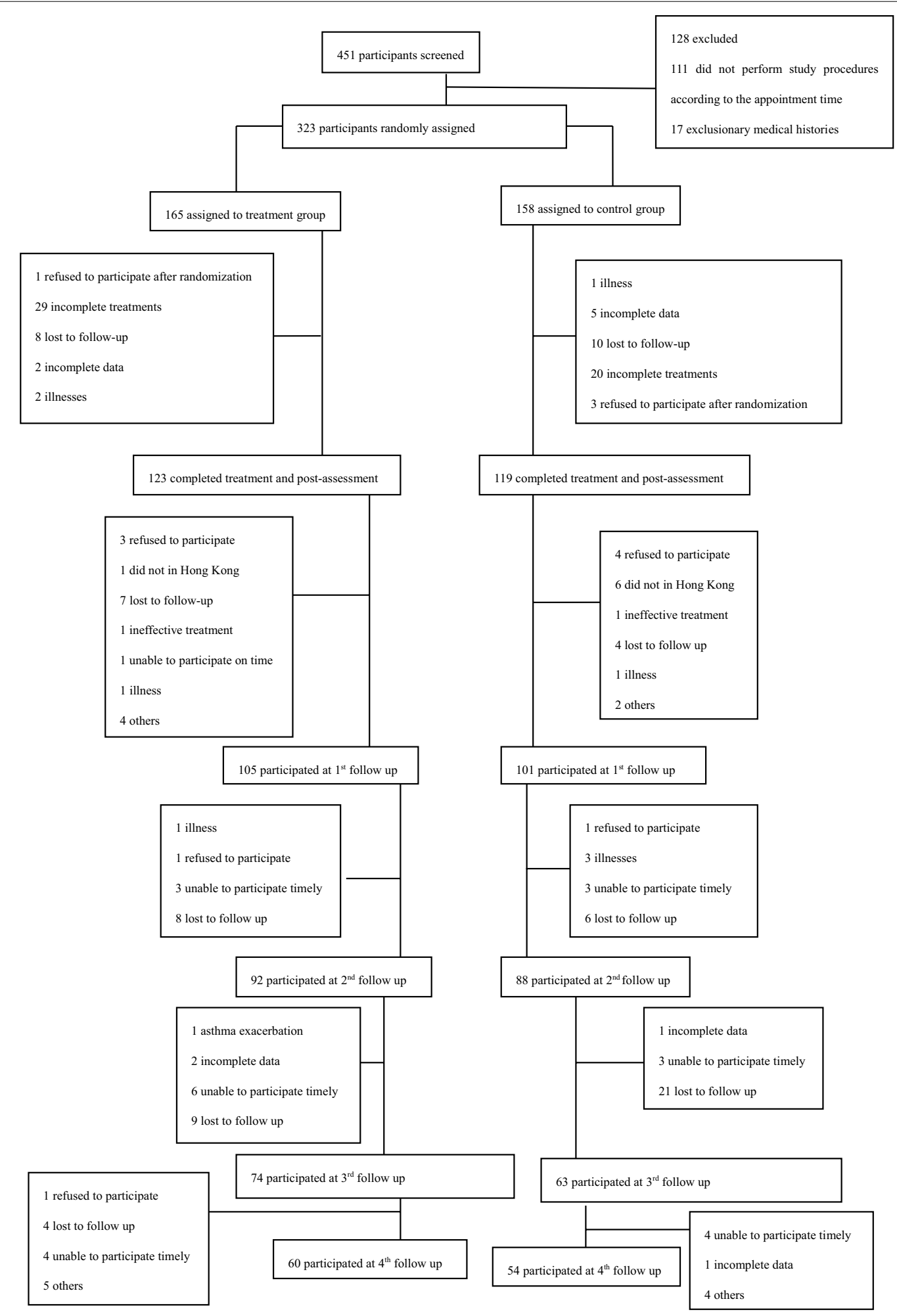

Fig. 5 Trial flow 
Table 1 Characteristics of study participants before and after treatment

\begin{tabular}{|c|c|c|c|c|c|c|}
\hline \multirow[t]{2}{*}{ Variable } & \multicolumn{3}{|l|}{ Before treatment } & \multicolumn{3}{|l|}{ After treatment } \\
\hline & $\begin{array}{l}\text { Acupoints herbal } \\
\text { patching mean (SD) }\end{array}$ & $\begin{array}{l}\text { Placebo } \\
\text { mean (SD) }\end{array}$ & $\mathbf{P}$ & $\begin{array}{l}\text { Acupoints herbal } \\
\text { patching mean (SD) }\end{array}$ & $\begin{array}{l}\text { Placebo mean } \\
\text { (SD) }\end{array}$ & $\mathbf{P}$ \\
\hline Age-year & $44.28 \pm 17.15$ & $44.53 \pm 17.83$ & 0.90 & & & \\
\hline \multicolumn{7}{|l|}{ Gender-no. (\%) } \\
\hline Male & $73(44.24)$ & $69(43.67)$ & 0.92 & & & \\
\hline Female & $92(55.76)$ & $89(56.32)$ & & & & \\
\hline Duration of asthma-year & $21.13 \pm 21.08$ & $22.74 \pm 22.68$ & 0.51 & & & \\
\hline $\begin{array}{l}\text { Asthma-related symptoms-no. of } \\
\text { days in previous } 1 \text { year }{ }^{\mathrm{a}}\end{array}$ & $10.25 \pm 12.26$ & $7.97 \pm 10.94$ & 0.08 & & & \\
\hline $\begin{array}{l}\text { Asthma-related symptoms-no. of } \\
\text { days during the treatment }\end{array}$ & & & & $2.58 \pm 0.47$ & $2.68 \pm 0.48$ & 0.88 \\
\hline \multicolumn{7}{|l|}{ Lung function } \\
\hline $\mathrm{FEV}_{1}$ & $2.13 \pm 1.08$ & $2.07 \pm 1.00$ & 0.57 & $2.04 \pm 0.41$ & $2.12 \pm 0.42$ & 0.14 \\
\hline $\mathrm{FEV}_{1}: \mathrm{FVC} \times 100$ & $85.97 \pm 13.85$ & $86.25 \pm 13.93$ & 0.86 & $87.36 \pm 0.66$ & $87.20 \pm 0.67$ & 0.87 \\
\hline \multicolumn{7}{|c|}{ Asthma-related health care use-no. (\%) } \\
\hline$\geq 1$ admit to $A \& E^{b}$ & $48(29)$ & $46(29)$ & 1.00 & $4(3)$ & $3(3)$ & 0.73 \\
\hline$\geq 1$ hospitalization & $26(16)$ & $29(18)$ & 0.54 & $3(2)$ & $2(2)$ & 0.68 \\
\hline$\geq 1$ outpatient visit & $134(81)$ & $134(84)$ & 0.39 & $13(12)$ & $14(12)$ & 0.77 \\
\hline$\geq 1$ prescription & $139(84)$ & $136(86)$ & 0.64 & $65(53)$ & $57(48)$ & 0.44 \\
\hline$\geq 1$ by own medication & $34(21)$ & $24(15)$ & 0.21 & $2(2)$ & $2(2)$ & 0.97 \\
\hline$\geq 1$ didn't need treatment & $26(16)$ & $17(11)$ & 0.19 & $8(7)$ & $6(5)$ & 0.63 \\
\hline \multicolumn{3}{|c|}{ The frequency of bronchodilator during asthma attack-no. (\%) } & 0.21 & & & 0.72 \\
\hline More than twice per day & $37(22)$ & $29(18)$ & & $13(11)$ & $13(11)$ & \\
\hline Once to twice per day & $50(30)$ & $62(39)$ & & $34(28)$ & $33(28)$ & \\
\hline 2-3 times per week & $12(7)$ & $18(11)$ & & $3(2)$ & $2(2)$ & \\
\hline Less than once per week & $48(29)$ & $36(23)$ & & $13(11)$ & $7(6)$ & \\
\hline Never & $18(11)$ & $13(8)$ & & $60(49)$ & $64(54)$ & \\
\hline $\begin{array}{l}\text { Symptoms which frequently } \\
\text { appeared in asthma participants- } \\
\text { no. of symptoms }\end{array}$ & $9.12 \pm 4.28$ & $9.22 \pm 4.18$ & 0.83 & $3.92 \pm 0.34$ & $4.01 \pm 0.37$ & 0.85 \\
\hline
\end{tabular}

Plus-minus value are mean \pm SD. P values for the comparison of means and percentages were calculated with the use of Chi-square test for categorical variables and the independent-samples test for continuous variables. FEV ${ }_{1}$, FVC

a The number of days with symptoms was calculated as the largest of the following variables during the previous 1 month: the number of days with wheezing, cough, chest tightness, or nighttime sleep disruption. This symptom scale ranges from 0-30 days

b Accident and emergency departments ( $A$ and $E$ )

c The total number of symptoms which frequently appeared in asthma participants are 23

automatically stopped after the 2nd acupoints herbal patching and had confirmed that has nothing to do with acupoints herbal patching, and one participants appeared the rash due to allergic to acupoints herbal patching patch. In addition, one participant has confirmed that she was pregnancy at the end of July 2010.

\section{Discussion}

In this study, participants with asthma in clinical remission stage who received acupoints herbal patching had no significant difference in primary and secondary outcomes at the end of 3rd treatment comparing with placebo group. Although ANCOVA show an insignificant effect of group $(\mathrm{P}>0.05)$, the effect of time was significant $(\mathrm{P}<0.001)$. This indicated that both groups experienced similar improvement, including significant decrease in the number of symptoms which were associated with Chinese Medicine, the percentage of participants with twenty-three symptoms which were associated with Chinese Medicine, the number of days with asthma related symptoms, etc. Only from the result of 3rd treatment, acupoints herbal patching seemed to be equal to the function of placebo, while rethinking its concept of acupoints herbal patching in Sanfu Days which means pasting herbal patch on some special acupoints in Sanfu Days. In others words, acupoints herbal patching in Sanfu Days 
Table 2 Characteristics of study participants' symptoms scale before and after treatment

\begin{tabular}{|c|c|c|c|c|c|c|}
\hline \multirow[t]{2}{*}{ Symptoms scale } & \multicolumn{3}{|l|}{ Before treatment } & \multicolumn{3}{|l|}{ After treatment } \\
\hline & $\begin{array}{l}\text { Acupoints herbal } \\
\text { patching }\end{array}$ & Placebo & $\mathbf{P}$ & $\begin{array}{l}\text { Acupoints herbal } \\
\text { patching }\end{array}$ & Placebo & $\mathbf{P}$ \\
\hline \multicolumn{7}{|l|}{ Detail symptoms-no. (\%) } \\
\hline 1. Wind intolerance & $61(37)$ & $67(42)$ & 0.32 & $13(11)$ & $13(11)$ & 0.93 \\
\hline 2. Susceptible cold & $88(53)$ & $96(61)$ & 0.09 & $16(13)$ & $10(8)$ & 0.25 \\
\hline 3. Sneeze, running nose before onset & $78(47)$ & $84(53)$ & 0.29 & $11(9)$ & $12(10)$ & 0.76 \\
\hline 4. Onset during quarter turn & $122(74)$ & $114(72)$ & 0.72 & $6(5)$ & $7(6)$ & 0.73 \\
\hline 5. Rapid or difficult breathing & $120(73)$ & $116(73)$ & 0.89 & $33(27)$ & $39(33)$ & 0.31 \\
\hline 6. Wake up by asthma symptoms & $113(68)$ & $110(70)$ & 0.83 & $29(24)$ & $23(19)$ & 0.42 \\
\hline 7. Bluish complexion & $24(15)$ & $17(11)$ & 0.31 & $4(3)$ & $1(1)$ & 0.19 \\
\hline 8. Spontaneous sweating & $52(32)$ & $56(35)$ & 0.45 & $6(5)$ & $10(8)$ & 0.27 \\
\hline 9. Lassitude & $95(58)$ & $86(54)$ & 0.57 & $20(16)$ & $20(17)$ & 0.91 \\
\hline 10. Lack of speech & $45(27)$ & $41(26)$ & 0.79 & $9(7)$ & $8(7)$ & 0.86 \\
\hline 11. Decline in physical strength & $79(48)$ & $66(42)$ & 0.27 & $19(15)$ & $12(10)$ & 0.21 \\
\hline 12. Reduction of exercise & $41(25)$ & $33(21)$ & 0.40 & $6(5)$ & $5(4)$ & 0.80 \\
\hline 13. Lack of strength & $91(55)$ & $90(57)$ & 0.74 & $23(19)$ & $17(14)$ & 0.36 \\
\hline 14. Lack of energy after asthma attack & $50(30)$ & $42(27)$ & 0.46 & $6(5)$ & $5(4)$ & 0.80 \\
\hline 15. Poor appetite & $31(19)$ & $25(16)$ & 0.48 & $4(3)$ & $7(6)$ & 0.33 \\
\hline 16. Abdomen fullness & $44(27)$ & $37(23)$ & 0.50 & $5(4)$ & $6(5)$ & 0.72 \\
\hline 17. Sloopy stool & $22(13)$ & $32(20)$ & 0.10 & $5(4)$ & $6(5)$ & 0.72 \\
\hline 18. Diarrhea after intake of oil food & $29(18)$ & $31(20)$ & 0.64 & $3(2)$ & $3(3)$ & 0.97 \\
\hline 19. Fear of cold & $71(43)$ & $72(46)$ & 0.65 & $15(12)$ & $12(10)$ & 0.60 \\
\hline 20. Soreness and weakness of waist and knees & $79(48)$ & $85(54)$ & 0.29 & $9(7)$ & $15(13)$ & 0.17 \\
\hline 21. Tinnitus & $60(36)$ & $54(34)$ & 0.68 & $10(8)$ & $11(9)$ & 0.76 \\
\hline 22. Frequent urination/night urination & $52(32)$ & $50(32)$ & 0.98 & $7(6)$ & $11(9)$ & 0.29 \\
\hline 23. Redness, hotness or anxiety & $58(35)$ & $53(34)$ & 0.76 & $9(7)$ & $7(6)$ & 0.65 \\
\hline
\end{tabular}

take effect in the treatment of asthma need three factors: the first is time (Sanfu Days), the second is meridian efficacy and the third is special herbal patch. In this study, placebo group also conducted in Sanfu Days and placebo patches were applied in same acupoints which means placebo group occupied two key factors. Hence, placebo group also can get an immediate improvement due to the psychological effect, time effect and meridian effect. So if only from the immediately result of both group at the end of 3rd treatment, there was nothing different occurred between acupoints herbal patching group and placebo group. On the whole, primary and secondary outcomes were insignificant different between treatment and control group, but the sub-analysis of secondary outcome in the 1st follow up show that the proportion of participants who did not need medical treatment when they had a small rise of asthma-related symptoms was $15 \%$ in acupoints herbal patching group better than $6 \%$ in placebo group $(\mathrm{P}<0.05)$. Winter and seasonal change was the peak time point for asthma attack in both groups according to the asthma attacks time distribution diagram of these 323 participants (Fig. 11). The 1st follow up was conducted in November in Hong Kong and it was a time from autumn to winter when asthma was easily attacked. It indicated that treatment group participants have a stronger resistance to asthma than placebo group during season change after one course acupoints herbal patching. Besides, acupoints herbal patching was a preventive treatment for asthma.

This study is, to date, the first RCT to compare acupoints herbal patching with placebo therapy for asthma in clinical remission stage. Its strengths included big sample size, long follow-up and strict blinding. Two inevitable limitations were the self-made questionnaire and imperfect blinding. There was no doubt that this study should adopt the international accepted questionnaire for clinical trial on asthma. But this project was full of Traditional Chinese Medicine Theory characteristic so it needed a questionnaire which combined Traditional Chinese Medicine with modern medicine well. So far, there was not related questionnaire that was the reason why this study designed the first questionnaire which owned the characteristic of both Traditional Chinese Medicine and modern medicine. This questionnaire was 


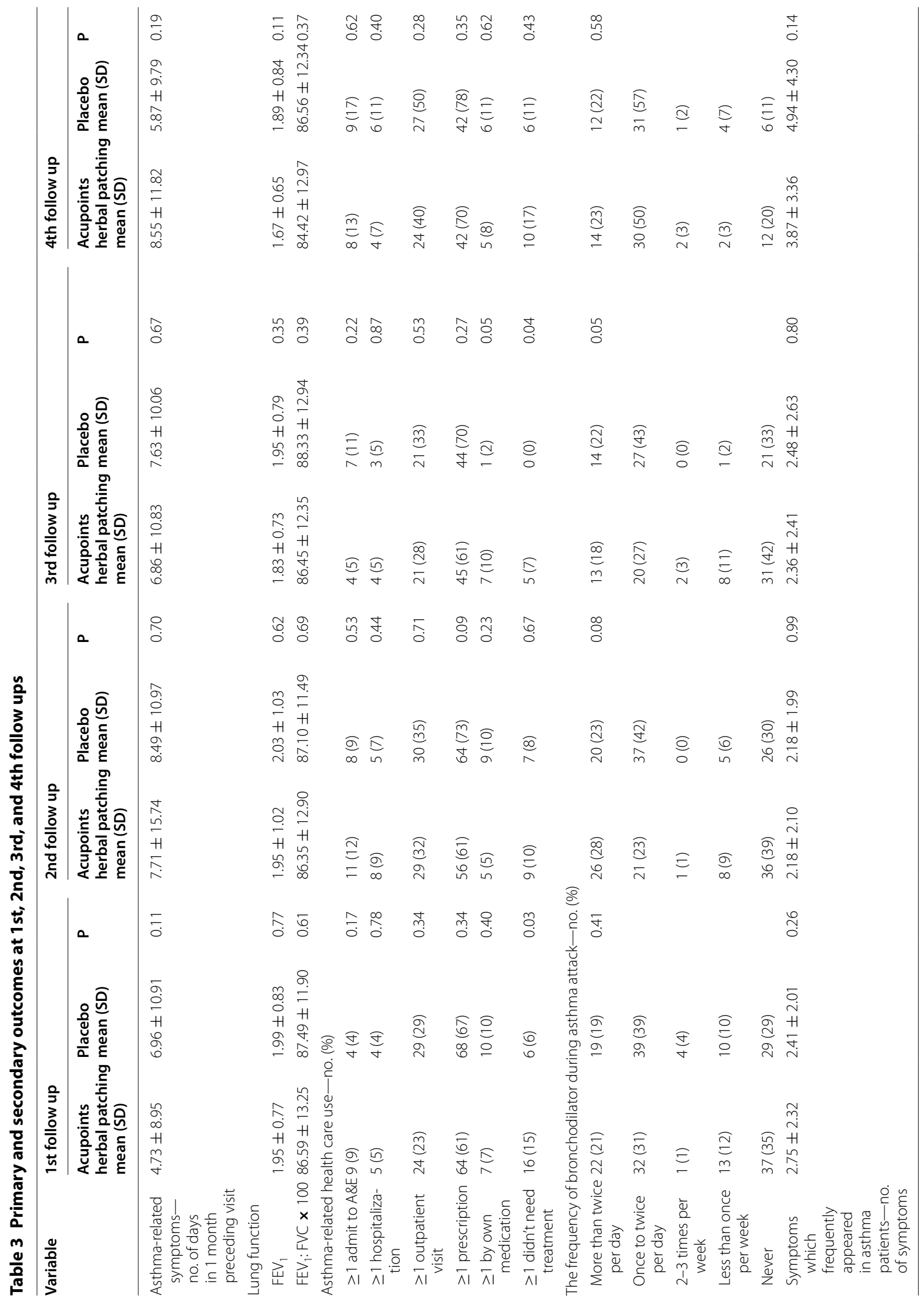


Table 4 Symptoms scale at 1st, 2nd, 3rd, and 4th follow ups

\begin{tabular}{|c|c|c|c|c|c|c|c|c|c|c|c|c|}
\hline \multirow{2}{*}{$\begin{array}{l}\text { Symptoms } \\
\text { scale }\end{array}$} & \multicolumn{3}{|c|}{ 1st follow up } & \multicolumn{3}{|c|}{ 2nd follow up } & \multicolumn{3}{|c|}{ 3rd follow up } & \multicolumn{3}{|c|}{ 4th follow up } \\
\hline & $\begin{array}{l}\text { Acupoints } \\
\text { herbal } \\
\text { patching }\end{array}$ & Placebo & $\mathbf{P}$ & $\begin{array}{l}\text { Acupoints } \\
\text { herbal } \\
\text { patching }\end{array}$ & Placebo & $\mathbf{P}$ & $\begin{array}{l}\text { Acupoints } \\
\text { herbal } \\
\text { patching }\end{array}$ & Placebo & $\mathbf{P}$ & $\begin{array}{l}\text { Acupoints } \\
\text { herbal } \\
\text { patching }\end{array}$ & placebo & $\mathbf{P}$ \\
\hline \multicolumn{13}{|c|}{ Detail symptoms-no. (\%) } \\
\hline $\begin{array}{l}\text { 1. Wind intoler- } \\
\text { ance }\end{array}$ & $13(12)$ & $13(13)$ & 0.92 & $9(10)$ & $4(5)$ & 0.18 & $5(7)$ & $6(10)$ & 0.55 & $12(20)$ & $7(13)$ & 0.31 \\
\hline $\begin{array}{l}\text { 2. Susceptible } \\
\text { cold }\end{array}$ & $18(17)$ & $17(17)$ & 0.95 & $18(20)$ & $19(22)$ & 0.74 & $9(12)$ & $12(19)$ & 0.27 & $15(25)$ & $20(37)$ & 0.16 \\
\hline $\begin{array}{l}\text { 3. Sneeze, } \\
\text { running nose } \\
\text { before onset }\end{array}$ & $14(13)$ & $12(12)$ & 0.76 & $7(8)$ & $16(18)$ & 0.03 & $8(11)$ & $8(13)$ & 0.73 & $12(20)$ & $18(33)$ & 0.11 \\
\hline $\begin{array}{l}\text { 4. Onset during } \\
\text { quarter turn }\end{array}$ & $19(18)$ & $15(15)$ & 0.53 & $11(12)$ & $14(16)$ & 0.44 & $7(9)$ & $12(19)$ & 0.11 & $19(32)$ & $20(37)$ & 0.55 \\
\hline $\begin{array}{l}\text { 5. Rapid or dif- } \\
\text { ficult breathing }\end{array}$ & $33(31)$ & $22(22)$ & 0.12 & $21(23)$ & $19(22)$ & 0.84 & $18(24)$ & $17(27)$ & 0.72 & $22(37)$ & $27(50)$ & 0.15 \\
\hline $\begin{array}{l}\text { 6. Wake up by } \\
\text { asthma symp- } \\
\text { toms }\end{array}$ & $22(21)$ & $23(23)$ & 0.75 & $13(14)$ & $24(27)$ & 0.03 & $7(9)$ & $9(14)$ & 0.38 & $13(22)$ & $20(37)$ & 0.07 \\
\hline $\begin{array}{l}\text { 7. Bluish com- } \\
\text { plexion }\end{array}$ & $0(0)$ & $1(1)$ & 0.31 & $0(0)$ & $2(2)$ & 0.15 & $2(3)$ & $1(2)$ & 0.66 & $2(3)$ & $3(6)$ & 0.56 \\
\hline $\begin{array}{l}\text { 8. Spontaneous } \\
\text { sweating }\end{array}$ & $4(4)$ & $7(7)$ & 0.32 & $2(2)$ & $6(7)$ & 0.13 & $0(0)$ & $5(8)$ & 0.01 & $5(8)$ & $6(11)$ & 0.62 \\
\hline 9. Lassitude & $18(17)$ & $10(10)$ & 0.13 & $11(12)$ & $8(9)$ & 0.53 & $12(16)$ & $7(11)$ & 0.40 & $16(27)$ & $12(22)$ & 0.58 \\
\hline $\begin{array}{l}\text { 10. Lack of } \\
\text { speech }\end{array}$ & $5(5)$ & $4(4)$ & 0.78 & $6(7)$ & $1(1)$ & 0.06 & $2(3)$ & $2(3)$ & 0.87 & $6(10)$ & $5(9)$ & 0.89 \\
\hline $\begin{array}{l}\text { 11. Decline } \\
\text { in physical } \\
\text { strength }\end{array}$ & $5(5)$ & $4(4)$ & 0.78 & $7(8)$ & $4(5)$ & 0.39 & $5(7)$ & $3(5)$ & 0.62 & $11(18)$ & $10(19)$ & 0.98 \\
\hline $\begin{array}{l}\text { 12. Reduction of } \\
\text { exercise }\end{array}$ & $4(4)$ & $0(0)$ & 0.05 & $2(2)$ & $0(0)$ & 0.16 & $1(1)$ & $2(3)$ & 0.47 & $5(8)$ & $3(6)$ & 0.56 \\
\hline $\begin{array}{l}\text { 13. Lack of } \\
\text { strength }\end{array}$ & $12(11)$ & $6(6)$ & 0.17 & $6(7)$ & $5(6)$ & 0.81 & $9(12)$ & $2(3)$ & 0.05 & $16(27)$ & $22(41)$ & 0.11 \\
\hline $\begin{array}{l}\text { 14. Lack of } \\
\text { energy after } \\
\text { asthma attack }\end{array}$ & $4(4)$ & $4(4)$ & 0.96 & $1(1)$ & $2(2)$ & 0.53 & $2(3)$ & $2(3)$ & 0.87 & $4(7)$ & $6(11)$ & 0.40 \\
\hline 15. Poor appetite & $5(5)$ & $4(5)$ & 0.78 & $3(3)$ & $2(2)$ & 0.69 & $2(3)$ & $3(5)$ & 0.52 & $5(8)$ & $4(7)$ & 0.86 \\
\hline $\begin{array}{l}\text { 16.abdomen } \\
\text { fullness }\end{array}$ & $8(8)$ & $5(5)$ & 0.43 & $4(4)$ & $1(1)$ & 0.19 & $3(4)$ & $0(0)$ & 0.11 & $4(7)$ & $4(7)$ & 0.88 \\
\hline 17. Sloopy stool & $3(3)$ & $2(2)$ & 0.69 & $3(3)$ & $2(2)$ & 0.69 & $2(3)$ & $5(8)$ & 0.17 & $3(5)$ & $8(15)$ & 0.08 \\
\hline $\begin{array}{l}\text { 18. Diarrhea after } \\
\text { intake of oil } \\
\text { food }\end{array}$ & $5(5)$ & $2(2)$ & 0.27 & $0(0)$ & $0(0)$ & 1.000 & $0(0)$ & $2(3)$ & 0.12 & $1(2)$ & $6(11)$ & 0.04 \\
\hline 19. Fear of cold & $10(10)$ & $7(7)$ & 0.50 & $5(5)$ & $7(8)$ & 0.50 & $10(14)$ & $5(8)$ & 0.30 & $8(13)$ & $11(20)$ & 0.31 \\
\hline $\begin{array}{l}\text { 20. Soreness } \\
\text { and weakness } \\
\text { of waist and } \\
\text { knees }\end{array}$ & $18(17)$ & $7(7)$ & 0.85 & $4(4)$ & $2(2)$ & 0.44 & $4(5)$ & $4(6)$ & 0.81 & $15(25)$ & $15(28)$ & 0.74 \\
\hline 21. Tinnitus & $12(11)$ & $8(8)$ & 0.40 & $4(4)$ & $7(8)$ & 0.31 & $4(5)$ & $1(2)$ & 0.24 & $12(20)$ & $12(22)$ & 0.77 \\
\hline $\begin{array}{l}\text { 22. Frequent uri- } \\
\text { nation /night } \\
\text { urination }\end{array}$ & 8 (8) & $8(8)$ & 0.94 & $5(5)$ & $1(1)$ & 0.11 & $2(3)$ & $4(6)$ & 0.30 & $8(13)$ & $6(11)$ & 0.72 \\
\hline $\begin{array}{l}\text { 23. Redness, hot- } \\
\text { ness or anxiety }\end{array}$ & $6(6)$ & $6(6)$ & 0.95 & $2(2)$ & $4(5)$ & 0.38 & $3(4)$ & $2(3)$ & 0.80 & $3(5)$ & $6(11)$ & 0.23 \\
\hline
\end{tabular}


Table 5 Temperature records of Sanfu Days in 2010 [22, 23]

\begin{tabular}{|c|c|c|c|c|c|}
\hline \multirow[t]{2}{*}{ Item time ${ }^{\text {ref }}$} & \multicolumn{5}{|l|}{2010} \\
\hline & $\begin{array}{l}\text { Maximum } \\
\text { temperature }\left({ }^{\circ} \mathrm{C}\right)\end{array}$ & $\begin{array}{l}\text { Temperature } \\
\left({ }^{\circ} \mathrm{C}\right)\end{array}$ & $\begin{array}{l}\text { Minimum } \\
\text { temperature }\left({ }^{\circ} \mathrm{C}\right)\end{array}$ & $\begin{array}{l}\text { Relative } \\
\text { humidity (\%) }\end{array}$ & $\begin{array}{l}\text { Sun exposure } \\
\text { (hour) }\end{array}$ \\
\hline 1st hottest day (July, 19) [22] & 33.6 & 29.7 & 27.3 & 77 & 10.9 \\
\hline 2nd hottest day (July, 29) [22] & 31.8 & 28.5 & 24.5 & 83 & 4.6 \\
\hline 3rd hottest day (Aug, 8) [23] & 32.2 & 28.8 & 25.8 & 84 & 7.4 \\
\hline
\end{tabular}
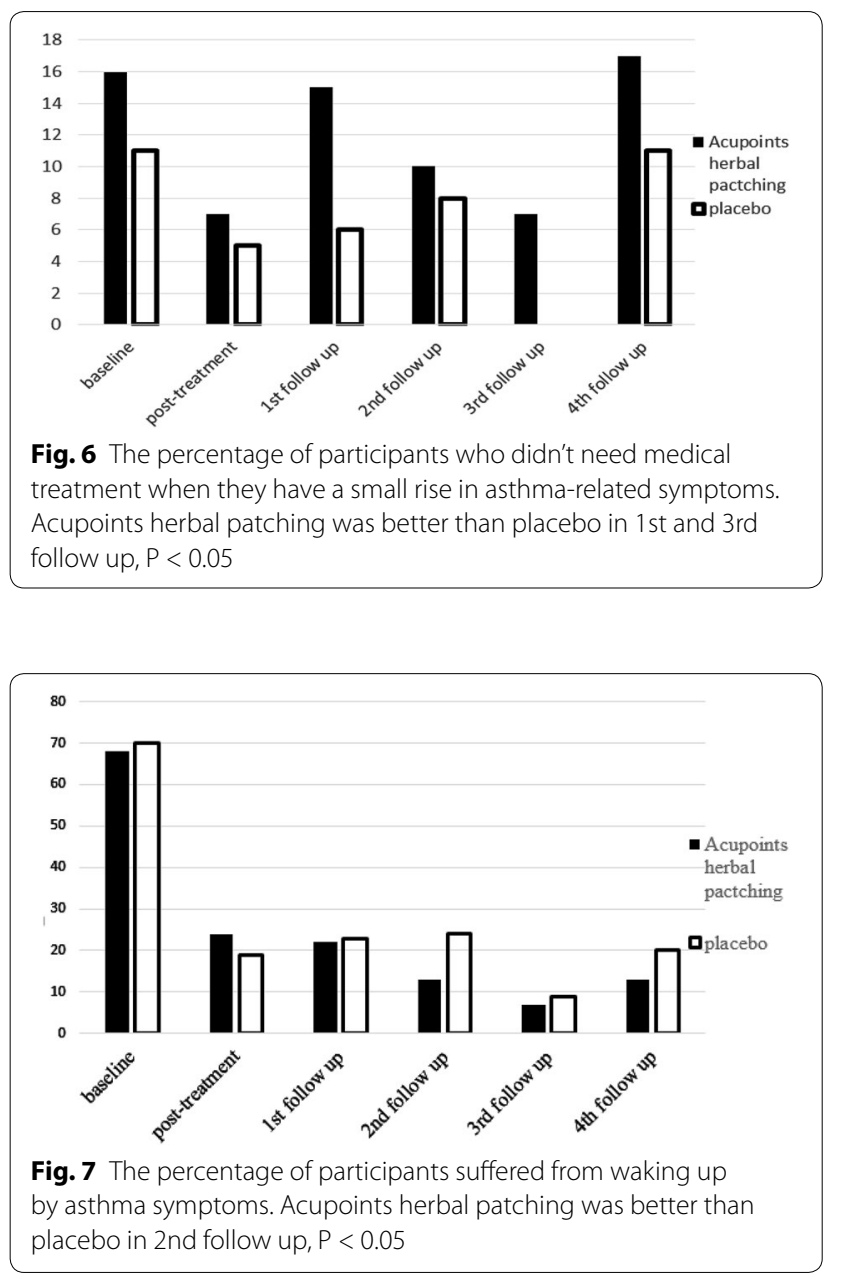

designed based on both Chinese Medicine and Western Medicine experts' consensus. Inevitably, this self-made questionnaire exist some shortcoming and the gap did exist between ideal and reality, such as this questionnaire only concerned about the asthma-related health care use when they had asthma-related symptoms, but didn't provide a section to record the detailed medications participants used, and usage and dosage. Hence, future study needed to further improve the quality of this self-made questionnaire and to detect its reliability and validity.
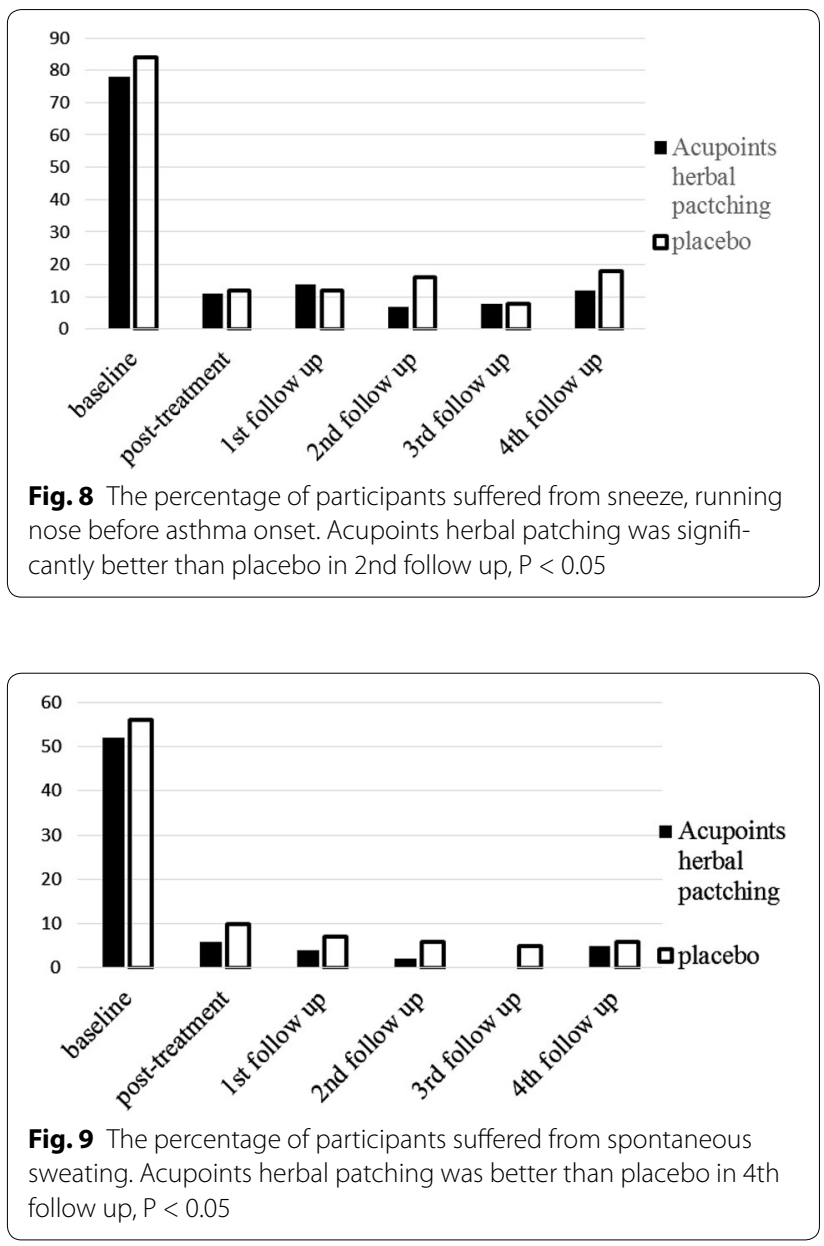

The second limitation was the imperfect blinding. Firstly, the smell of real and placebo herbal patch was different, even participants didn't have the chance to see the patches, we cannot stop patches' odor emitted; Secondly, participants in acupoints herbal patching group inevitably suffered much greater skin irritations, which will easily lead to the failure of blinding when participants from real acupoints herbal patching group communicated with placebo group; Thirdly, even participants have no idea of acupoints herbal patching before they entering this study, after joining this study, they might become 


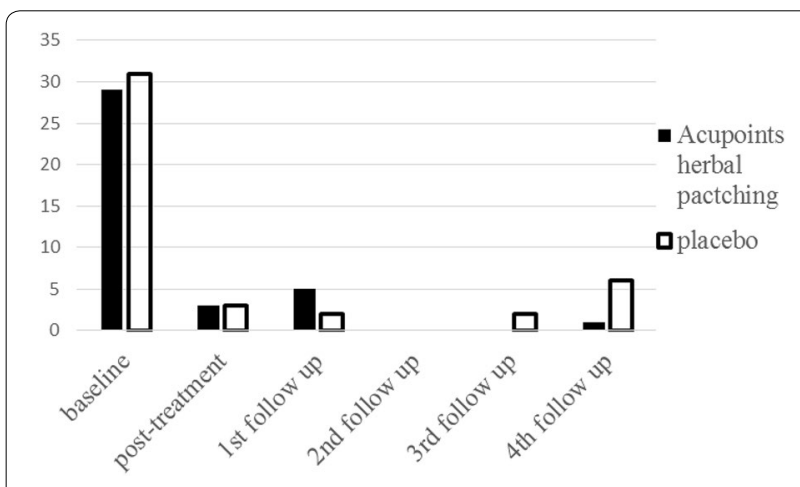

Fig. 10 The percentage of participants susceptibly suffered from diarrhea after intake of oily food. Acupoints herbal patching was better than placebo in 4th follow up, $P<0.05$

interested in acupoints herbal patching and try to learn more about acupoints herbal patching. When participants know something about acupoints herbal patching, they might be easily aware that herbal patches consist of irritated ingredients which could incur skin irritations. Although blinding existed some inevitable limitations, its merits were still worth to be mentioned: firstly, herbal patch appearance look almost like placebo patch; secondary patch applied in the same 11 acupoints; thirdly, same duration and both in Sanfu Days. Even the Chinese Medicine practitioners have no idea about which group they conducted before unblinding. The nearly same drop-out rate in post-treatment and four times follow ups support that the blinding of this study was relatively successful. And after treatment, both groups got a significant improvement comparing with the baseline. All these show that placebo effect did exist during treatment and most participants in placebo group believed that they had received the real treatment. $\beta$-agonists, which are the most common drug treatment during acute asthma attack while are associated with various adverse effects $[4,5]$. In this study, the statistical results showed that the proportion of participants who didn't need to use pharmaceutical treatment when they have a small rise in asthma-related symptoms was significantly increased after acupoints herbal patching. Besides, adverse events in this study were very minor, and the morbidity of side-effects in acupoints herbal patching group was insignificant higher than placebo group. TCM considerate that Sanfu Days are the three hottest in a year and both Yang-qi of body and nature are most exuberant in these days. From the temperature record of 2010, it was found that the average temperature in Sanfu Days from July 19, 29 and August 8 in 2010 (Table 5) was pretty high, all of them were above $31{ }^{\circ} \mathrm{C}$, air humidity was above $77 \%$, and sunshine duration was more than $4.6 \mathrm{~h}[25,26]$. Hence, acupoints herbal patching in Sanfu days might be recommend as a complementary preventive treatment for chronic stable asthma especially for Qi-deficiency and Yang-deficiency patients.

\section{Conclusions}

There was no significant difference between acupoints herbal patching and placebo group at the end of 3rd treatment and four times follow ups in pulmonary function test. The result was different from Li YH's clinical studies about acupoints herbal patching for asthma, which show that acupoints herbal patching can significantly improve participants' lung function [27]. Also, the secondary outcome of acupoints herbal patching group was no significant difference as compare with placebo group. However, the sub-analysis of secondary outcomes in the four times follow ups show that as time goes on, the lasting effect of acupoints herbal patching was superior to placebo especially in improving Lung-qi-deficiency and

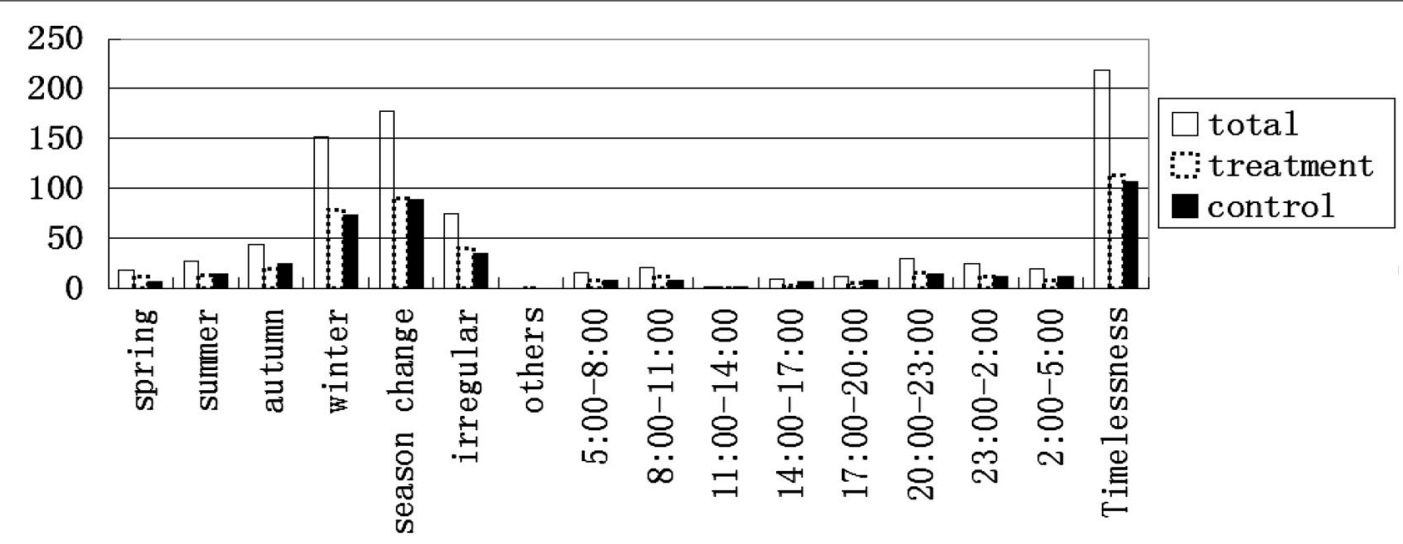

Fig. 11 Asthma attack time distribution diagram 
Spleen-qi-deficiency syndromes. What's more, the lasting effect of acupoints herbal patching was better than placebo in reducing medication need when they had a small rise in asthma-related symptoms. Acupoints herbal patching will not only be a significant alternative treatment with a low risk of side effect for asthma participants, but also provide a good choice for health policy makers.

\begin{abstract}
Abbreviations
1st: first; 2nd: second; 3rd: third; 4th: fourth; FEV1: forced expiratory volume in one second; FEV1/FVC (\%): forced expiratory volume in one second/forced vital capacity; ITT: intention-to-treat; PP: per-protocol; SPSS: statistical Package for the Social Sciences; ANCOVA: analysis of covariance; FVC: forced vital capacity; SD: standard deviation; TG: treatment group; CG: control group; Nov: November; Feb: February; Ref: reference; TCM: traditional chinese medicine; $\mathrm{RCT}$ : randomized control trial; IRB: institutional review board.
\end{abstract}

\section{Authors' contributions}

LZ and LL did substantial contributions to the conception, design of the work, the acquisition, analysis, and interpretation of data for the work; Besides, they drafted and revised the work; Lastly, they were agreed to be accountable for all aspects of the work in ensuring that questions related to the accuracy and integrity of any part of the work are appropriately investigated and resolved. Also, WZ, VW, ZE did substantial contributions to the conception of the work; And they drafted the work for important intellectual content; Finally, they were agreed to be accountable for all aspects of the work in ensuring that questions related to the accuracy or integrity of any part of the work are appropriately investigated and resolved. KL, WC, TY and LL contributed much to the acquisition and analysis of data for the work; And they revised the work critically for important intellectual content; Lastly, they were agreed to be accountable for all aspects of the work in ensuring that questions related to the accuracy or integrity of any part of the work are appropriately investigated and resolved. All authors read and approved the final manuscript.

\section{Author details \\ ${ }^{1}$ School of Chinese Medicine, The University of Hong Kong, 10 Sassoon Road, Pokful, Hong Kong, China. ${ }^{2}$ Chinese Medicine Department, Hospital Authority, Hong Kong, China. ${ }^{3}$ Center of Reproductive Medicine and Constitu- tion of Traditional Chinese Medicine, Beijing University of Chinese Medicine, Beijing, China.}

\section{Acknowledgements}

The study was financially supported by grant from Hong Kong Hospital Authority's funding for Chinese Medicine (Project Code: 200006030).The authors are grateful for the support of all student helpers, office staffs and research assistants from the school of Chinese Medicine, the University of Hong Kong. The sponsors have nothing to do with study design, data collection and analysis, decision to publish, or preparation of the manuscript.

\section{Competing interests}

The authors declare that they have no competing interests.

Part of this article was presented in abstract form at the 2014 ICACI on "International Conference on Allergy and Clinical Immunology," May 26-27, 2014, London, United Kingdom.

Received: 1 June 2015 Accepted: 25 January 2016

Published online: 04 February 2016

\section{References}

1. Tattersfield AE, Knox AJ, Britton JR, Hall IP (2002) Asthma. Lancet 360:1313-1322
2. Taylor DR, Drazen JM, Herbison GP, Yandava CN, Hancox RJ, Town GI (2000) Asthma exacerbations during long term-agonist use: influence of 2-adrenoceptor polymorphism. Thorax 55:762-767

3. Spitzer WO, Suissa S, Ernst $P$ et al (1992) The use of $\beta$-agonists and the risk of death and near death from asthma. N Engl J Med 326:501-506

4. Greening AP, Ind PW, Northfield M, Shaw G (1994) Added salmeterol versus higher-dose corticosteroid in asthma participants with symptoms on existing inhaled corticosteroid. Allen and Hanburys Limited UK Study Group. Lancet 344:219-224

5. O'Byrne PM, Barnes PJ, Rodriguez-Roisin R et al (2001) Low dose inhaled budesonide and formoterol in mild persistent asthma. Am J Respir Crit Care Med 164:1392-1397

6. Pauwels RA, Löfdahl CG, Postma DS et al (1997) Effect of inhaled formoterol and budesonide on exacerbations of asthma. Formoterol and Corticosteroids Establishing Therapy (FACET) International Study Group. N Engl J Med 337:1405-1411

7. Wong CA, Walsh LJ, Smith CJP et al (2000) Inhaled corticosteroid use and bone-mineral density in participants with asthma. Lancet 355:1399-1403

8. Israel E, Banerjee TR, Fitzmaurice GM, Kotlov TV, LaHive K, LeBoff MS (2001) Effects of inhaled glucocorticoids on bone density in premenopausal women. N Engl J Med 345:941-947

9. Cumming RG, Mitchell P, Leeder SR (1997) Use of inhaled corticosteroids and the risk of cataracts. N Engl J Med 337:8-14

10. Garbe E, LeLorier J, Boivin JF (1997) Inhaled and nasal Glucocorticoids and the risk of occular hypertension or open-angled glaucoma. JAMA 277:722-727

11. Zeng EX, Chen YJ (2006) Effect observations of natural moxibustion therapy in bronchus asthma. Mod Nurs 12:412-413

12. Lai XS, Li YM, Zhang JW (2000) The influence of acupoints herbal patching to sIL-2R and T-cell of asthma participants. Chin Acupunct Moxib $1: 22-25$

13. Dai WJ, Lai XS (2001) The effect of medicinal vesiculation therapy on plasma SP and VIP contents in bronchial asthma participants. Acupunct Res 26:124-127

14. Lu WZ (2007) The impact of acupoints application on cytokines IL-4, IL-10 and IL-12 in asthma participants. Liaoning J Tradit Chin Med 24:120-129

15. Meng Y, Cai SX (2001) IL-12 and its role in the mechanism of asthma attack. J First Military Med Univ 9:711

16. Zhang $\mathrm{JL}$ (2002) The polymorphisms of IL-4 and IL-10 gene promoter region and asthma. Foreign Med Sci (Sect Immunol) 2:104

17. Yang JJ, Lai XS (2002) Research on the efficacy and mechanism of acupoints herbal patching for bronchial asthma. Jilin J Traditi Chin Med 22:7-8

18. Chen M, Lu XL, Zhen OR, Xu W (2005) Research on the relationship between the efficacy of acupoints herbal patching in Sanfu Days and ET. IgE Lung Funct 18:44-46

19. Nagase T, Fukuchi Y, Jo C (1990) Endothelin-1 stimulates arachidonate 15-lipoxygenase activity and oxygen radical formation in the rat distal lung. Biochem Biophys Res Commun 168(2):485-489

20. Yang HZ, Yao X (2013) Effect observation of acupoint herbal patching with Yifei capsule for bronchial asthma. Chin J Chin Med Inf 20:71-72

21. Ma H, Xiao XT, Zheng FR (2009) Effect observation of self-prescription combined with acupoint herbal patching in Sanfu Days for bronchial asthma in remission stage. Clin J Tradit Chin Med 21:537-538

22. Liang YJ, Que DM (2010) Acupoint herbal patching in Sanfu Days with in sanjiu days for 50 case of asthma in remission stage. Jiangxi J Tradit Chin Med 41:69-70

23. Liu RQ (2007) SPSS software. Chinese TCM Publishing House, Beijing

24. Li LX, Gao YH, Zhang PD et al (2007) Generalized linear mixed models and its application. Mod Prev Med 34:2103-2104

25. Hong Kong observatory. Daily extract of meteorological observations, July 2010. 2010. http://www.weather.gov.hk/cis/dailyExtract_e. $h t m ? y=2010 \& m=7$. Accessed 21 Sept 2015

26. Hong Kong observatory. Daily extract of meteorological observations, August 2010. 2010. http://www.weather.gov.hk/cis/dailyExtract_ uc.htm?y=2010\&m=8. Accessed 21 Sept 2015

27. Li YY, Song WD, Ju YL, Mo JM, Zhu T (2009) Research on the impact of acupoints herbal patching on the lung function of asthma participants. J New Med 19:101-102 